\title{
The Missing Science: Epidemiological data gaps for COVID-19 policy in the United States
}

Rajiv Bhatia ${ }^{1}$ *, Isabella Sledge, Stefan Baral ${ }^{2}$

1 Primary Care and Population Health, Stanford University, Stanford, CA

2 Department of Epidemiology, Johns Hopkins School of Public Health, Baltimore, MD

* Corresponding author: drajiv@stanford.edu

\section{Abstract}

We report on a scoping study of COVID-19 epidemiological data available for COVID-19 policy and management decisions for U.S. settings. We synthesize current US Centers for Disease and Control and Prevention (CDC) estimates for parameter of infectious transmission, infection severity, and disease burden, and summarize epidemiologic contributions to these parameters published by CDC-affiliated investigators through Oct 30, 2020. Authoritative estimates of most infectious transmission and infection severity parameters exist but rely primarily on data from studies conducted in non-U.S. populations. Estimates of secondary infection risks for household, workplace, school, or other community settings are lacking and estimates of the clinical fraction remain uncertain. The CDC reports multiple disease incidence and prevalence measures at national and state geographies, including some measures disaggregated by age group, race/ethnicity and sex; however, nationally uniform disease burden measures are not available at the sub-state level or for sub-populations defined by exposure setting, limiting opportunities for targeted interventions. CDC-affiliated investigators authored 133 quantitative studies on COVID-19 through Oct 30, 2020; however only 34 employed analytic methods. The remainder were descriptive. Of the 34 analytic studies, eleven reported on risk factors for infection, seven reported on risk factors for severe disease, three on symptomatic infections, three reported secondary infection risks, and four reported on indirect pandemic effects. Gaps remain in the epidemiological data required 
medRxiv preprint doi: https://doi.org/10.1101/2021.02.11.21251602; this version posted February 12, 2021. The copyright holder for this preprint (which was not certified by peer review) is the author/funder, who has granted medRxiv a license to display the preprint in perpetuity. It is made available under a CC-BY-NC-ND 4.0 International license .

for an efficient and equitable public health policy response to COVID-19. The existence of these gaps one year after the onset of the COVID-19 pandemic underscores the need for standardizing data collection and research priorities and protocols in the context of a rapidly emerging infectious disease epidemics.

Word Count: 3830

Tables: 3

Supplemental Materials 


\section{Introduction}

In any emerging infectious disease epidemic, efficient and defensible disease control interventions will require timely and complete epidemiological data. The scope and severity of the US COVID-19 epidemic and its enormous social costs makes the need for actionable epidemiologic data particularly acute. Yet, one year after the discovery of the SARS-CoV-2 virus, the US Centers for Disease Control (CDC) acknowledges that "information about the biological aspects of SARS-CoV-2 and epidemiological characteristics of COVID-19 remain limited...." [1] Insufficient data on transmission mechanisms, setting and activity specific risks, and population-specific disease burden may have reduced the effectiveness, efficiency, and equity of the public health response. [2]

An established literature on emerging respiratory virus epidemics provides a framework for evaluating the adequacy of Covid-19 data required for policy and management decisions. Lessons learned from prior epidemics including H1N1 influenza (H1N1), Severe Adult Respiratory Syndrome (SARS-CoV-1), and Middle-East Respiratory Syndrome (MERS) all inform CDC pandemic plans for emerging influenza viruses. The CDC's pandemic interval framework [3] and the pandemic severity assessment framework [4] explicitly spell out the scientific and data needs for assessing the risks posed by emerging respiratory viral infections. Reviews critically examining the epidemiology of the H1N1 pandemic response elaborate on these data demands, $[5,6,7,8]$ which were re-iterated at the onset of the COVID-19 pandemic. $[9,10]$

A scoping study is a method to examine the extent, range, nature of evidence or research activities in a particular field and to identify research gaps. [11] Here, we report on a scoping study of COVID-19 epidemiologic parameters for infectious transmission, infection severity, and disease burden which, collectively, inform the threat level posed by an emerging respiratory virus epidemic and guide an efficient, proportional and equitable public health response. [8]. We first synthesize current authoritative federal government appraisals for each of these parameters. We then summarize the methods and outcomes of epidemiologic investigations published by CDC-affiliated investigators informing the aforementioned parameters. We focus specifically on CDC appraisals and investigations because of the 
medRxiv preprint doi: https://doi.org/10.1101/2021.02.11.21251602; this version posted February 12, 2021. The copyright holder for this preprint (which was not certified by peer review) is the author/funder, who has granted medRxiv a license to display the preprint in perpetuity. It is made available under a CC-BY-NC-ND 4.0 International license .

agency's unique access to the data required for COVID-19 epidemiology, its partnerships with state and local public health authorities, and its leadership role in pandemic management. We identify knowledge gaps and suggest opportunities to improve the evidence base for COVID-19 mitigation.

\section{METHODS}

Table 1 summarizes the parameters for infectious transmission, disease severity, and disease burden that we sought along with their typical data sources. 
Table 1. Epidemiological data required for emerging respiratory virus epidemic management

\begin{tabular}{|c|c|c|c|}
\hline Measure & Definition & Information value & Typical source \\
\hline \multicolumn{4}{|c|}{ Infectious Transmission } \\
\hline $\begin{array}{l}\text { Basic reproductive } \\
\text { number }\end{array}$ & $\begin{array}{l}\text { The expected number of secondary } \\
\text { cases directly generated by one } \\
\text { case }\end{array}$ & $\begin{array}{l}\text { Potential speed of } \\
\text { epidemic growth }\end{array}$ & $\begin{array}{l}\text { Calculated from contact rate, } \\
\text { secondary infection risk, and } \\
\text { infectious period or from the } \\
\text { growth rate of the early disease } \\
\text { incidence curve }\end{array}$ \\
\hline Growth rate & $\begin{array}{l}\text { Change per unit time (acceleration } \\
\text { or deceleration) of the incidence rate }\end{array}$ & $\begin{array}{l}\text { Current trajectory of } \\
\text { epidemic growth }\end{array}$ & Population disease monitoring \\
\hline $\begin{array}{l}\text { Fraction of the } \\
\text { population susceptible }\end{array}$ & $\begin{array}{l}\text { Proportion of the population who do } \\
\text { not have immunity to the infection or } \\
\text { to disease }\end{array}$ & $\begin{array}{l}\text { Targeting control } \\
\text { measures }\end{array}$ & $\begin{array}{l}\text { Studies of immunity such as } \\
\text { seroprevalence of antibodies }\end{array}$ \\
\hline Incubation period & $\begin{array}{l}\text { Interval between infection and the } \\
\text { development of symptoms }\end{array}$ & $\begin{array}{l}\text { Timeframe for prevention } \\
\text { of secondary infection }\end{array}$ & Transmission studies \\
\hline $\begin{array}{l}\text { Duration of } \\
\text { infectiousness }\end{array}$ & $\begin{array}{l}\text { Viral load and duration in } \\
\text { symptomatic and } \\
\text { asymptomatic people }\end{array}$ & $\begin{array}{l}\text { Timeframe for prevention } \\
\text { of secondary infection }\end{array}$ & Transmission studies \\
\hline Serial interval & $\begin{array}{l}\text { Interval between development of } \\
\text { symptoms in a case and an infected } \\
\text { contact }\end{array}$ & $\begin{array}{l}\text { Necessary for capturing } \\
\text { the } R_{0}\end{array}$ & Transmission studies \\
\hline $\begin{array}{l}\text { Fraction of pre- } \\
\text { symptomatic } \\
\text { transmission }\end{array}$ & $\begin{array}{l}\text { Proportion of infections spread by } \\
\text { persons who appear well but are } \\
\text { infected and later develop symptoms }\end{array}$ & $\begin{array}{l}\text { Timeframe for prevention } \\
\text { of secondary infection }\end{array}$ & Transmission studies \\
\hline $\begin{array}{l}\text { Secondary infection } \\
\text { risk (SIR or } \\
\text { alternatively, } \\
\text { secondary attack rate }\end{array}$ & $\begin{array}{l}\text { Proportion of exposed people who } \\
\text { become ill particular setting } \\
\text { (household, school workplace) }\end{array}$ & $\begin{array}{l}\text { Targeting control } \\
\text { measures }\end{array}$ & Transmission studies \\
\hline \multicolumn{4}{|c|}{ Infection Severity } \\
\hline Clinical fraction & $\begin{array}{l}\text { Proportion of infected people who } \\
\text { become ill }\end{array}$ & $\begin{array}{l}\text { Predicting number of } \\
\text { transmitted infections that } \\
\text { will result in illness; } \\
\text { adopting a proportional } \\
\text { response }\end{array}$ & $\begin{array}{l}\text { Household or contact tracing } \\
\text { transmission studies }\end{array}$ \\
\hline $\begin{array}{l}\text { Case or infection } \\
\text { hospitalization ratios }\end{array}$ & $\begin{array}{l}\text { How many people who become } \\
\text { infected require hospitalization }\end{array}$ & $\begin{array}{l}\text { Predicting the scale and } \\
\text { scope of health care } \\
\text { services; adopting a } \\
\text { proportional response }\end{array}$ & $\begin{array}{l}\text { Population surveillance } \\
\text { Cohort studies } \\
\text { Large transmission studies }\end{array}$ \\
\hline $\begin{array}{l}\text { Case or infection } \\
\text { fatality ratio }\end{array}$ & How many infected people die & $\begin{array}{l}\text { Adopting a proportional } \\
\text { response }\end{array}$ & $\begin{array}{l}\text { Reported hospital data. Death } \\
\text { records }\end{array}$ \\
\hline Severity risk factors & $\begin{array}{l}\text { Risk factors, including ethnicity, } \\
\text { income, comorbidities, healthcare } \\
\text { access, social characteristics, } \\
\text { environmental conditions, affecting } \\
\text { vulnerability to disease }\end{array}$ & $\begin{array}{l}\text { Targeting control } \\
\text { measures }\end{array}$ & $\begin{array}{l}\text { Case control studies } \\
\text { Syndromic surveillance }\end{array}$ \\
\hline \multicolumn{4}{|c|}{ Disease Burden } \\
\hline Incidence rate & $\begin{array}{l}\text { Number of new cases of illness, } \\
\text { hospitalization or death in a } \\
\text { population per unit time }\end{array}$ & $\begin{array}{l}\text { Is the disease } \\
\text { accelerating or slowing } \\
\text { down and where }\end{array}$ & $\begin{array}{l}\text { Syndromic surveillance } \\
\text { Serial prevalence studies } \\
\text { Disease hospitalization rates } \\
\text { Disease mortality rates }\end{array}$ \\
\hline $\begin{array}{l}\text { Point or period } \\
\text { prevalence }\end{array}$ & $\begin{array}{l}\text { Proportion of the population that is a } \\
\text { current case a point or period in time }\end{array}$ & $\begin{array}{l}\text { Current level of active } \\
\text { infection and transmission }\end{array}$ & Symptom and test-based surveys \\
\hline $\begin{array}{l}\text { Community attack } \\
\text { rate / cumulative } \\
\text { incidence }\end{array}$ & $\begin{array}{l}\text { Number of new cases of disease } \\
\text { during specified time interval }\end{array}$ & $\begin{array}{l}\text { Population disease } \\
\text { burden \& remaining } \\
\text { population susceptible }\end{array}$ & $\begin{array}{l}\text { Cohort studies, statistical } \\
\text { estimation }\end{array}$ \\
\hline
\end{tabular}


medRxiv preprint doi: https://doi.org/10.1101/2021.02.11.21251602; this version posted February 12, 2021. The copyright holder for this preprint (which was not certified by peer review) is the author/funder, who has granted medRxiv a license to display the preprint in perpetuity.

It is made available under a CC-BY-NC-ND 4.0 International license .

Transmission studies, which observe individuals with confirmed infection and the people they infect secondarily, provide data on the mechanism of transmission (e.g. person to person through respiratory secretions), the incubation period (how long after infection symptoms appear), the generation interval (the time between a person becoming infected and subsequently infecting someone else), the infectivity period (how long an infected person can spread the illness), and the risk of infection among exposed contacts in various settings (secondary infectious risks/secondary attack rates). Transmission studies also identify populations relatively more susceptible to infection as well as groups or settings relatively more likely to contribute to transmission. [12] Additionally, transmission studies can quantify presymptomatic transmission and the proportion of infections that are symptomatic (the clinical fraction). These latter two parameters will, in part, determine the feasibility of isolation and contact tracing strategies. $[13,14]$ Optimal understanding of transmission and an equitable mitigation response requires that studies include diverse community settings (e.g., household, workplace, school, and congregate living such as long-term care facilities and shelters).

Infection severity measures include estimates of the proportion of infected individuals who require hospitalization or who die. Estimates of infection severity depend upon probable and confirmed case definitions and thus on the surveillance systems that ascertain infection and infection-related morbidity and death. Severity measures are vulnerable to many biases and may vary by place and over time as surveillance methods, case definitions, testing strategies, clinical understanding, and prevention efforts evolve. Cohort studies including large case ascertained studies (where households are enrolled after an index case is identified) with adequate follow up can provide reliable estimates of disease severity measures in addition to informing transmission risks. [8]

Disease burden is the product of infection transmission and severity contextualized by socio-demographic and environmental risk factors and their disparities. Measures of disease burden include the incidence and prevalence of illness, numbers of people hospitalized, and infection-related deaths. Systematically monitoring health care visits for infection-related illness is one reliable indicator of the disease burden if such surveillance is combined with routine testing of a representative sample of patients visits. [8] 
Optimally, data on the incidence of infection-related illness should be available in real time and disaggregated by population subgroup, setting, severity and patient characteristics to inform timely, targeted community mitigations and anticipation of healthcare needs.

The CDC's Pandemic Severity Assessment Framework (PSAF), an element of the national pandemic strategy, integrates the aforementioned transmission, severity and disease burden parameters into a qualitative assessment of the threat posed by an emerging infection. [4] Such risk assessments should be updated as the epidemic evolves, and data improves. Table S1 (Supplemental Materials) illustrates CDC's application of the PSAF for 2009 Influenza H1N1. [4]

We accessed public CDC webpages (See list in supplemental Materials, Table S2) to find current federal estimates of SARS-CoV-2 transmission, infection severity, and disease burden parameters and their data sources. We also searched specifically for documents containing applications of pandemic risk assessment tools, such as the PSAF.

To evaluate original contributions to COVID-19 epidemiology by CDC-affiliated investigators, we first reviewed all studies in the collection of COVID-19 research in the Morbidity and Mortality Weekly Report (MMWR)—a principal vehicle for publishing studies conducted by CDC investigators and their state partners-as well as the CDC COVID-19 Science update webpage. We further searched PubMed for studies with COVID-19 in the title or abstract, a medical subject heading (MeSH) for epidemiology, and CDC as an affiliation. Additionally, we examined the COVID-19 research collections in Emerging Infectious Disease, Clinical Infections Disease (CID), the Journal of the American Medical Association (JAMA), and the New England Journal of Medicine (NEJM). We included studies published from 1 Jan to 30 Oct 2020. We excluded studies that did not present quantitative data.

We classified each included study as either descriptive or analytic and then abstracted published information on study design, data source, setting, population, and outcomes. (Supplemental Materials, Table S3) We considered a study descriptive if it did not evaluate or formally test a hypothesis using a 
well-established epidemiological method. We classified studies that quantified risk of infection, disease severity, or transmission through an established epidemiological method as analytic. If a study reported both descriptive and analytic components, we classified it as analytic.

We subcategorized descriptive studies by their design as: case study, case series, cluster or outbreak investigation, cross-sectional or ecologic survey. We categorized the designs of analytic studies as either cross-sectional, case-control, ecologic prospective cohort, or retrospective cohort designs. We identified each study's data source as either passive, sentinel, syndromic, or active surveillance programs, administrative program records, medical records, or survey instruments. We also categorized each study with regards to its setting and population.

We abstracted study outcomes as reported by the investigators. For each study other than those in the case studies and the case series, cluster and outbreak categories investigations, we identified outcomes contributing to any of the key aforementioned epidemiologic parameters.

\section{RESULTS}

Table 2 synthesizes CDC's current estimates of epidemiologic parameters for SARS-CoV-2 transmission, infection severity and disease along with the estimates referenced sources. 
medRxiv preprint doi: https://doi.org/10.1101/2021.02.11.21251602; this version posted February 12, 2021. The copyright holder for this preprint (which was not certified by peer review) is the author/funder, who has granted medRxiv a license to display the preprint in perpetuity.

\section{It is made available under a CC-BY-NC-ND 4.0 International license .}

Table 2. CDC estimates for SARS-CoV-2 infection transmissibility, severity and disease burden measures

\begin{tabular}{|c|c|c|c|c|c|}
\hline Measures & Estimate (range) & Estimate date & CDC Webpage & $\begin{array}{c}\text { Sources referenced } \\
\text { by CDC }\end{array}$ & $\begin{array}{l}\text { Referenced source } \\
\text { study setting(s) }\end{array}$ \\
\hline \multicolumn{6}{|c|}{ Transmissibility } \\
\hline $\begin{array}{l}\text { Reproductive } \\
\text { number }\end{array}$ & $2.5(2.0-4.0)$ & 10 Sep 2020 & $\frac{\frac{\text { COVID-19 }}{\text { Pandemic }}}{\frac{\text { Planning }}{\text { Scenarios[15] }}}$ & Li et al. [16] & Multiple settings \\
\hline Susceptibility & $100 \%$ & 10 Sep 2020 & $\begin{array}{l}\frac{\text { COVID-19 }}{\text { Pandemic }} \\
\frac{\text { Planning }}{\text { Scenarios [15] }}\end{array}$ & Not sourced & \\
\hline $\begin{array}{l}\text { Mean incubation } \\
\text { period }\end{array}$ & $\sim 6$ days (mean) & 10 Sep 2020 & $\begin{array}{l}\frac{\text { COVID-19 }}{\text { Pandemic }} \\
\text { Planning } \\
\text { Scenarios [15] }\end{array}$ & McAloon et al.[17] & China \\
\hline $\begin{array}{l}\text { Mean serial } \\
\text { interval }\end{array}$ & $\sim 6$ days (mean) & 10 Sep 2020 & $\begin{array}{l}\frac{\text { COVID-19 }}{\text { Pandemic }} \\
\frac{\text { Planning }}{\text { Scenarios [15] }}\end{array}$ & He et al.[18] & China \\
\hline $\begin{array}{l}\text { Percentage of } \\
\text { transmission } \\
\text { occurring prior to } \\
\text { symptom onset }\end{array}$ & $50 \%(30-70)$ & 10 Sep 2020 & $\begin{array}{l}\frac{\text { COVID-19 }}{\text { Pandemic }} \\
\frac{\text { Planning }}{\text { Scenarios [15] }}\end{array}$ & $\begin{array}{l}\text { He et al.[18]; Casey et } \\
\text { al.[19] }\end{array}$ & China, Northern Italy \\
\hline $\begin{array}{l}\text { Duration of } \\
\text { infectiousness }\end{array}$ & $\begin{array}{l}<10 \text { days after } \\
\text { symptom onset }\end{array}$ & 19 Oct 2020 & $\begin{array}{l}\text { Duration of } \\
\text { Isolation and } \\
\text { Precautions for } \\
\text { Adults with } \\
\text { COVID-19 [20] }\end{array}$ & & \\
\hline $\begin{array}{l}\text { Secondary } \\
\text { infection risk } \\
(\mathrm{SIR}) \\
\end{array}$ & No estimates & & & & \\
\hline \multicolumn{6}{|c|}{ Infection Severity } \\
\hline $\begin{array}{l}\text { Percent of } \\
\text { infections that } \\
\text { are } \\
\text { asymptomatic }\end{array}$ & $40 \%(10-70 \%)$ & 10 Sep 2020 & $\begin{array}{l}\frac{\text { COVID-19 }}{\text { Pandemic }} \\
\frac{\text { Planning }}{\text { Scenarios [15] }}\end{array}$ & $\begin{array}{l}\text { Byambasuren et al. } \\
\text { [21]; Poletti et al.[89 ] }\end{array}$ & Multiple settings \\
\hline $\begin{array}{l}\text { Infection } \\
\text { hospitalization } \\
\text { ratio }\end{array}$ & No estimates & & & & \\
\hline $\begin{array}{l}\text { Infection fatality } \\
\text { ratio }\end{array}$ & $\begin{array}{c}0-19 \text { years: } 0.00003 ; \\
20-49 \text { years: } 0.0002 ; \\
50-69 \text { years: } 0.005 ; \\
70+\text { years: } 0.054\end{array}$ & 10 Sep 2020 & $\begin{array}{l}\frac{\text { COVID-19 }}{\text { Pandemic }} \\
\frac{\text { Planning }}{\text { Scenarios [15] }}\end{array}$ & Hauser et al.[22] & European Union \\
\hline $\begin{array}{l}\text { Hospital fatality } \\
\text { ratio }\end{array}$ & $\begin{array}{c}18-49 \text { years: } 2.4 \% ; 50- \\
64 \text { years: } 10.0 \% ; \geq 65 \\
\text { years: } 26.6 \%\end{array}$ & 10 Sep 2020 & $\begin{array}{l}\frac{\text { COVID-19 }}{\text { Pandemic }} \\
\frac{\text { Planning }}{\text { Scenarios [15] }}\end{array}$ & COVID-NET [23] & USA, 13 States \\
\hline
\end{tabular}


medRxiv preprint doi: https://doi.org/10.1101/2021.02.11.21251602; this version posted February 12, 2021. The copyright holder for this preprint (which was not certified by peer review) is the author/funder, who has granted medRxiv a license to display the preprint in perpetuity.

It is made available under a CC-BY-NC-ND 4.0 International license .

\begin{tabular}{|c|c|c|c|c|c|}
\hline $\begin{array}{l}\text { Rate ratio for } \\
\text { hospitalization } \\
\text { by age }\end{array}$ & $\begin{array}{l}\text { 0-4 years: } 4 x \text { lower } \\
\text { 5-17 years: } 9 x \text { lower } \\
\text { 18-29: comparison } \\
\text { 30-39 years: } 2 x \text { higher } \\
\text { 40-49 years: } 3 x \text { higher } \\
\text { 50-64 years: } 4 x \text { higher } \\
\text { 65-74 years: } 5 x \text { higher } \\
\text { 75-84 years: } 8 x \text { higher } \\
\text { 85+ years: } 13 x \text { higher }\end{array}$ & 18 Aug 2020 & $\begin{array}{l}\frac{\text { COVID-19 }}{\text { Hospitalization }} \\
\text { and Death by } \\
\underline{\text { Age }[24]}\end{array}$ & COVID-NET [23] & USA, 13 States \\
\hline $\begin{array}{l}\text { Rate ratio for } \\
\text { fatality by age }\end{array}$ & $\begin{array}{c}\text { 0-4 years: } 9 x \text { lower } \\
\text { 5-17 years: } 16 x \text { lower } \\
\text { 18-29: comparison } \\
\text { 30-39 years: } 4 x \text { higher } \\
\text { 40-49 years: } 10 x \\
\text { higher } \\
\text { 50-64 years: } 30 x \\
\text { higher } \\
\text { 65-74 years: } 90 x \\
\text { higher } \\
\text { 75-84 years: } 220 x \\
\text { higher } \\
\text { 85+ years: } 630 x \\
\text { higher }\end{array}$ & 18 Aug 2020 & $\frac{\frac{\text { COVID-19 }}{\text { Hospitalization }}}{\frac{\text { and Death by }}{\text { Age [24] }}}$ & $\begin{array}{l}\text { National Center for } \\
\text { Health Statistics [25] }\end{array}$ & USA, 50 States \\
\hline $\begin{array}{l}\text { Rate ratio for } \\
\text { confirmed case } \\
\text { by race / } \\
\text { ethnicity }\end{array}$ & $\begin{array}{c}\text { American Indian or } \\
\text { Alaska Native, 1.8x; } \\
\text { Asian, 0.6x; Black, } \\
\text { 1.4x; Latino, 1.7x }\end{array}$ & 30 Nov 2020 & $\begin{array}{l}\frac{\text { COVID-19 }}{\text { Hospitalization }} \\
\text { and Death by } \\
\text { Race/Ethnicity } \\
\text { [26] }\end{array}$ & & USA, 50 States \\
\hline $\begin{array}{l}\text { Rate ratio for } \\
\text { hospitalization } \\
\text { by race / } \\
\text { ethnicity }\end{array}$ & $\begin{array}{c}\text { American Indian or } \\
\text { Alaska Native, 4.0x; } \\
\text { Asian, 1.2x; Black, } \\
\text { 3.7x; Latino, 4.1x }\end{array}$ & 30 Nov 2020 & $\begin{array}{l}\frac{\text { COVID-19 }}{\text { Hospitalization }} \\
\text { and Death by } \\
\text { Race/Ethnicity } \\
{[26]}\end{array}$ & COVID-Net [23] & USA, 13 States \\
\hline $\begin{array}{l}\text { Rate ratio for } \\
\text { fatality by race / } \\
\text { ethnicity }\end{array}$ & $\begin{array}{c}\text { American Indian or } \\
\text { Alaska Native, 2.6x; } \\
\text { Asian, 1.1x; Black, } \\
\text { 2.8x; Latino, 2.8x }\end{array}$ & 30 Nov 2020 & $\begin{array}{l}\frac{\text { COVID-19 }}{\text { Hospitalization }} \\
\frac{\text { and Death by }}{\text { Race/Ethnicity }} \\
26]\end{array}$ & $\begin{array}{l}\mathrm{NCHS} \text { provisional } \\
\text { death counts [27] }\end{array}$ & USA, 50 States \\
\hline \multicolumn{6}{|c|}{ Disease burden } \\
\hline $\begin{array}{l}\text { Share of } \\
\text { ambulatory visits } \\
\text { for influenza like } \\
\text { illness }\end{array}$ & $0.7-3.3 \%$ & 2021, week 4 & CovidView [28] & $\begin{array}{l}\text { Influenza-like-illness } \\
\text { Network [29] }\end{array}$ & USA \\
\hline $\begin{array}{l}\text { Share of } \\
\text { emergency } \\
\text { department visits } \\
\text { for coronavirus- } \\
\text { like-illness }\end{array}$ & $5.1 \%$ & 2021, week 4 & CovidView [30] & $\begin{array}{l}\text { National Syndromic } \\
\text { Surveillance Program } \\
\text { (NSSP) [31] }\end{array}$ & USA, 47 States \\
\hline $\begin{array}{l}\text { SARS-CoV-2 } \\
\text { laboratory-test- } \\
\text { associated } \\
\text { hospital } \\
\text { admissions } \\
\end{array}$ & $7.6 / 100,000$ persons & 2021, week 4 & CovidView [32] & COVID-NET [32] & USA, 13 regions \\
\hline $\begin{array}{l}\text { Percentage of } \\
\text { deaths attributed } \\
\text { to pneumonia, } \\
\text { influenza, or } \\
\text { COVID-19 (PIC) }\end{array}$ & $28.4 \%$ & 2021, week 4 & CovidView [33] & $\begin{array}{l}\text { National Center for } \\
\text { Vital Statistics [34] }\end{array}$ & USA, 50 States \\
\hline $\begin{array}{l}\text { SARS-CoV-2 } \\
\text { antibody } \\
\text { seroprevalence }\end{array}$ & $1.4-24 \%$ & Dec 2020 & $\frac{\text { Nationwide }}{\text { Commercial }}$ & CDC & USA, 49 States \\
\hline
\end{tabular}




\begin{tabular}{|c|c|c|c|c|c|}
\hline & & & $\begin{array}{l}\text { Seroprevalence } \\
\text { Survey [35] }\end{array}$ & & \\
\hline $\begin{array}{l}\text { Cumulative } \\
\text { attack rate }\end{array}$ & $\begin{array}{c}25,412 / 100,000 \\
\text { persons }\end{array}$ & 19 Jan 2021 & $\begin{array}{l}\text { Estimated } \\
\text { Disease Burden } \\
\text { of COVID-19 } \\
{[36]}\end{array}$ & CDC & USA, 50 States \\
\hline
\end{tabular}

CDC reports authoritative estimates of most aforementioned infection transmission parameters with the exception of setting-specific secondary infection risks. The majority of CDC transmission data estimates have relied on epidemiological studies conducted outside the U.S.

With regards to disease severity, CDC estimates the asymptomatic fraction as $40 \%$ but with a wide range $(10-70 \%)$ based on summary estimates from meta-analytic reviews as well as individual studies. [1] CDC estimated case fatality and case hospitalization ratios in May 2020 but later removed these parameters. [37] In September 2020, CDC adapted infection fatality ratios from an analysis of European data. [1]

CDC estimates risk ratios for hospitalization and death for age groups and race/ethnicity based on data from the COVID-NET active surveillance program. [38] No estimates of relative risks for infection, hospitalization or death exist for occupations or particular community activities or settings.

Since April 2020, CDC has reported several COVID-19 disease incidence measures on a weekly basis, including emergency department visits for coronavirus-like illness (CLI), SARS-CoV-2 associated hospital admissions, and deaths from pneumonia, influenza, and COVID-19 [39]. CDC additionally reports some of these incidence measures at the level of states or multi-state regions. CLI emergency department visits represent those for fever plus cough and/or shortness of breath or difficulty breathing and come from National Syndromic Surveillance Program (NSSP) that collects and integrates data on ED visit symptoms from a large share of the countries emergency department in 47 states. CDC's COVID-NET program conducts active surveillance for laboratory-confirmed SARS-CoV-2 hospitalizations in 13 participating sub-state regions publishing age-stratified incidence rates weekly. CDC reports the percentage of deaths attributed to pneumonia or influenza as well as weekly counts of COVID-19 attributed deaths for each 
state parsed by age, gender, and race / ethnicity. Total excess deaths by cause of death is also available for each state. [40]

In July 2020, CDC reported seroprevalence estimates from 10 sites based on COVID-19 antibodies detected in serum samples collected by commercial laboratories for reasons other than COVID-19. [41] After Aug 2020, CDC expanded seroprevalence reporting to all U.S. states and has been reporting estimates every two weeks. In December 2020, CDC provided the first estimates of cumulative prevalence of infection and symptomatic infection at a national level. [42]

CDC affiliated investigators published 133 epidemiologic studies through Oct 30, 2020. (Supplemental materials Table S3) Of these 133 studies, 99 were descriptive and 34 analytic. Table 3 tabulates study characteristics for both groups of studies. 
medRxiv preprint doi: https://doi.org/10.1101/2021.02.11.21251602; this version posted February 12, 2021. The copyright holder for this preprint (which was not certified by peer review) is the author/funder, who has granted medRxiv a license to display the preprint in perpetuity.

It is made available under a CC-BY-NC-ND 4.0 International license .

Table 3. Published epidemiologic studies on COVID-19 transmission, infection severity, and disease burden authored by CDC affiliated investigators

\begin{tabular}{|c|c|c|}
\hline & $\begin{array}{l}\text { Descriptive Studies } \\
\qquad \mathrm{N}=99\end{array}$ & $\begin{array}{c}\text { Analytic Studies } \\
\qquad N=34\end{array}$ \\
\hline \multicolumn{3}{|l|}{ Publication month } \\
\hline Feb & 3 & 0 \\
\hline Mar & 4 & 1 \\
\hline Apr & 12 & 1 \\
\hline May & 12 & 1 \\
\hline Jun & 7 & 6 \\
\hline Jul & 18 & 5 \\
\hline Aug & 17 & 3 \\
\hline Sep & 9 & 9 \\
\hline Oct & 17 & 8 \\
\hline \multicolumn{3}{|l|}{ Publication } \\
\hline Clinical Infectious Diseases & 1 & 7 \\
\hline Emerging Infectious Diseases & 1 & 0 \\
\hline JAMA & 1 & 0 \\
\hline Morbidity and Mortality Weekly Report & 92 & 27 \\
\hline New England Journal of Medicine & 4 & 0 \\
\hline \multicolumn{3}{|l|}{ Study Design } \\
\hline Case series, cluster, or outbreak & 31 & \\
\hline Case study & 3 & \\
\hline Cross Sectional & 43 & 17 \\
\hline Case-control & & 2 \\
\hline Ecologic & 25 & 8 \\
\hline Prospective Cohort & & 4 \\
\hline Retrospective Cohort & & 3 \\
\hline Study Data Source & & \\
\hline
\end{tabular}


medRxiv preprint doi: https://doi.org/10.1101/2021.02.11.21251602; this version posted February 12, 2021. The copyright holder for this preprint (which was not certified by peer review) is the author/funder, who has granted medRxiv a license to display the preprint in perpetuity.

\section{It is made available under a CC-BY-NC-ND 4.0 International license .}

\begin{tabular}{|c|c|c|}
\hline Field Data Collection & 38 & 10 \\
\hline Medical Records & 2 & 3 \\
\hline Active Surveillance & 10 & 1 \\
\hline Passive Surveillance & 37 & 7 \\
\hline Sentinel Surveillance & 2 & \\
\hline Syndromic Surveillance & 3 & 1 \\
\hline Survey & 3 & 11 \\
\hline National Vital Statistics System & 0 & 2 \\
\hline Other & 2 & 1 \\
\hline \multicolumn{3}{|l|}{ Study Setting } \\
\hline Childcare & 2 & 0 \\
\hline College or university & 1 & 1 \\
\hline Correctional or detention facility & 7 & 0 \\
\hline Cruise ship & 2 & 0 \\
\hline General community setting & 60 & 27 \\
\hline Healthcare facility & 3 & 1 \\
\hline Homeless shelter & 2 & 1 \\
\hline Industrial & 2 & 1 \\
\hline Military & 1 & 1 \\
\hline Youth program & 2 & 0 \\
\hline Private gathering & 4 & 0 \\
\hline Public gathering & 2 & 0 \\
\hline Skilled nursing & 11 & 2 \\
\hline \multicolumn{3}{|l|}{ Study sub-population } \\
\hline Athletes & 1 & 1 \\
\hline Children & 12 & 0 \\
\hline College students & 1 & 0 \\
\hline General population & 52 & 26 \\
\hline Healthcare workers & 5 & 0 \\
\hline Homeless individuals & 3 & 1 \\
\hline Incarcerated individuals & 5 & 0 \\
\hline Industrial workers & 3 & 1 \\
\hline
\end{tabular}


medRxiv preprint doi: https://doi.org/10.1101/2021.02.11.21251602; this version posted February 12,2021 . The copyright holder for this preprint (which was not certified by peer review) is the author/funder, who has granted medRxiv a license to display the preprint in perpetuity.

\section{It is made available under a CC-BY-NC-ND 4.0 International license .}

\begin{tabular}{|l|c|c|}
\hline LCTF residents & 11 & 2 \\
\hline Military personnel & 1 & 1 \\
\hline Pregnant women & 1 & 0 \\
\hline Travelers & & \\
\hline & & 3 \\
\hline Analytic outcomes ${ }^{1}$ & & 11 \\
\hline Secondary Infection Risks & & 3 \\
\hline Effect measure of risk factor for infection & & 7 \\
\hline Proportion of symptomatic infections & & \\
\hline Effect measures for risk factors for & & \\
\hline hospitalization, ICU stay, or death & 5 & 0 \\
\hline Incidence measures for infection, illness, & & \\
\hline hospitalization, or death & & \\
\hline Indirect pandemic effects & & \\
\hline
\end{tabular}

${ }^{1}$ All studies classified except those with case study or case series design 
Of the 99 descriptive studies we identified, thirty-one were case series, 43 were cross-sectional, and 25 were ecologic. Most descriptive studies summarized laboratory confirmed cases of SARS-CoV-2 infection from a particular setting, disaggregating counts by clinical outcomes, by patient demographic or medical attributes, by exposure type, or by temporal patterns. Descriptive studies reported data from a wide range of populations and community settings.

Some descriptive studies postulated mechanisms of transmission or prevention; however, they did not estimate quantitatively infection transmission or disease severity parameters. Sixteen of the descriptive studies reported on the incidence or prevalence of laboratory confirmed infection, clinical illness, hospitalization or death in either the general population or a defined subpopulation (e.g., skilled nursing facility residents, healthcare workers, incarcerated or homeless individuals) for a defined time period. [43, $44,45,46,47,41,48,49,50,51,52,53,54,55,56,57]$. One study provided a point estimate of excess deaths associated with COVID-19 using national vital statistics data. [57]

We classified thirty-four studies as analytic. Most (31/34) were published after June 2020 and in the publication MMWR (27/34). The remainder were published in other peer-reviewed journals. Most of the analytic studies had general population subjects (27/34), had cross-sectional (17/34) or ecologic (8/34) designs, and utilized field (10/34) or survey data (11/24).

Most analytic studies estimated at least one parameter for transmission, infection severity, or disease burden. Of the analytic studies estimating transmission parameters, three estimated secondary infection risks (SIR) $[58,59,60]$ One of these was a follow up study of the first 12 confirmed cases in the US [58] Two others estimated the SIR for household contacts $[59,60]$. Our searches found no studies estimating the SIR for workplace, school or community settings or for particular occupations or industries.

Eleven analytic studies provided estimates of an effect measure for risk factors for laboratory-confirmed infections. $[59,61,62,63,64,65,66,67,68,69,70]$ Five of the eleven were conducted using a sample of the general population. These examined the frequency or particular demographics, [61] neighborhood deprivation/social vulnerability levels, $[63,68]$ relationship to a confirmed case, [59] and recent social 
activities, including restaurant dining. [66] Other investigations examined various factors related to confirmed infection in sub-populations, including face coverings and distancing among occupants of a military ship, [62] ethnic composition of employees of industrial facilities, [64] shelter residence status among people experiencing homelessness, [69] screening strategies and staffing levels in skilled nursing facilities. [65,67] Eight of the eleven studies employed cross-sectional or ecologic designs. No studies examined the independent effects of etiologic variables in multi-variate analysis.

Seven studies examined risk factors for severe disease outcomes, including hospitalization, ICU stay and death. Most of these used general population subjects. [52, 63,71, 72, 73, 74,75$]$ These studies consistently found older age to be a strong predictor of need for ICU stays, mechanical ventilation and of death. Male gender, end-stage renal disease, coronary artery disease, and neurologic disorders, immunocompromising conditions, pregnancy, African American identity were also correlated with severe disease. $[71,72,73,52,75]$ One study estimated the odds of prolonged symptoms in relation to disease severity. [74]

No CDC-affiliated studies estimated infection-hospitalization or infection-fatality ratios. Three studies assessed symptom prevalence or the fraction of symptomatic people among individuals with positive tests. One study, conducted in a military setting, found that $18.5 \%(44 / 238)$ of those with positive tests were asymptomatic. [62] Two community studies found that nearly all household contacts of an index case had symptoms or developed them shortly after testing positive. [76,77]

Nine analytic studies reported on indirect health impacts of COVID-19, including impacts on emergency departments visits, trends in childhood vaccination, telehealth visits, workplace absenteeism, mental health symptoms, and characteristics associated with delay or avoidance of medical care. $[78,79,80,81$, $82,83,84,85,86$ ] One found declines in emergency care visits for heart attacks, strokes, and diabetes; [81] one found an increased prevalence of mental health symptoms; [85] one found increased absenteeism in non-healthcare essential workers; [82] and two documented an initial decline followed by a rebound in childhood vaccination rates. [78,83] 


\section{DISCUSSION}

Twelve months after the identification of the SARS-COV-2 virus, epidemiologic understanding of infectious transmission, disease severity, and disease burden has evolved and advanced. However, despite a substantial number of original epidemiological investigations, our review finds data gaps which may limit efficient interventions to prevent COVID-19 transmission.

We restricted this scoping study to epidemiological studies authored or co-authored by CDC investigators. Thus, our review may have also overlooked data found in pre-prints and other unpublished analyses. However, estimating transmission, infection severity and disease burden parameters generally require detailed individual case records or contact tracing data for which access is restricted to government agencies.

\section{Infectious Transmission}

CDC's current estimates of SARS-CoV-2 transmission parameters, including the incubation period, serial interval, and timing and duration of infectiousness, come from studies conducted outside the U.S. yet are consistent across settings and time-periods and thus considered reliable. On the other hand, CDC estimates of the clinical fraction have a wide range, reflecting significant heterogeneity among study methods, settings and populations. $[87,88,89,90]$ Large or multi-regional case-ascertained studies or examination of contact tracing follow up data conducted in the US could provide more precise and agespecific estimates of this parameter as well as of the impact of asymptomatic spread in different settings.

Context-specific estimates of the SIR, including for schools, workplaces, and other community settings, remain unavailable. These parameters require large or setting-specific transmission studies. Available estimates of the SIR for household transmission also vary widely with few based on US settings. No identified transmission studies have quantitatively examined the efficacy of modifying exposure factors within households or workplaces. 
Current guidance for COVID-19 community mitigations relies primarily on indirect evidence. For example, pre-symptomatic transmission, proximity, and an inability to mask are the facts used to justify restrictions on indoor dining in restaurants; [91] Such restrictions may well be protective; however, rigorous studies quantifying transmission risks for restaurant settings have not been undertaken.

Studies comparing estimates of the SIR across community settings and activities could identify high risk activities and prioritize risk reduction interventions. Large coordinated case-ascertained prospective studies in particular could be a valuable tool to determine risks from specific activities in household, community, business, transportation, and educational settings and assess the risk reduction associated with operating rules for interrupting transmission. Such studies could inform the relative population-level impacts of social distancing, indoor versus outdoor settings, infectivity of high-touch surfaces, and the value of indoor ventilation. The state-to-state variation in policy response also provides natural experiments to evaluate the relative effectiveness of mandatory and voluntary community mitigations measures.

While several case series and outbreak investigations have highlighted potential hazards in "essential" and frontline work settings such as food production, no identified analytic studies quantified determinants of risks of transmission in workplace settings. Case control studies examining comparative risks across industries and occupations could contribute to understanding how occupational exposures contribute, both directly and through household exposures, to COVID-19 racial disparities.

Studies authored by CDC investigators during the 2009 influenza H1N1 pandemic illustrate the value of timely, robust transmission epidemiology. During 2009, CDC investigators conducted a number of influential transmission studies within the first nine months of the onset of the H1N1 epidemic. One, conducted on the basis of state-submitted case reports in the first months of the epidemic, found that odds ratios for $\mathrm{H} 1 \mathrm{~N} 1$ infection among household contacts were 3.5 times $(1.6-8.0)$ higher for 0-4 year old and 2 times $(1.1$ - 3.6) higher for 5-18 years old than for 19-50 year old individuals. [92] Another CDC study combined data from 7 household transmission studies early in the 2009 pandemic to quantify the 
timing of secondary infections within households, demonstrating that about $5 \%$ happen $>3$ days after onset of symptoms. [93] A meta-analysis of 47 studies on transmission rates in households, schools, workplaces, and social events, including fifteen in settings within the U.S., identified multiple population determinants of secondary infection risks, including higher risks for students relative to teachers and for boarding versus day schools. [94]

\section{Disease Severity}

CDC's estimates of the infection-fatality ratios (IFR) based on studies in six European countries may not be generalizable to the US. County to country differences in the prevalence of vulnerability factors, medical care, and ascertainment of infection affect this parameter. Community cohort studies could provide assessment of multiple infection severity parameters.

While the CDC has established older age as a strong risk factor for severe COVID-19 disease and characterized the relative risk associated with individual medical co-morbidities, [95] further inquiry could examine how the effects of age and co-morbidities vary for individuals with different levels of functional status and in different residential settings (e.g., skilled nursing, assisted living or independent community living).

\section{Burden of infection and disease}

To monitor disease burden, the CDC has routinely reported several measures of infection and disease incidence at the national and state level; however, these measures have limited geographical resolution. Each state has utilized its own distinct sets of state and regional indicators, [96] along with state-specific benchmarks, to guide control activities.

For calibrating mitigations, most states have relied heavily on laboratory-test-based "case" counts, which underestimate infection rates and vary depending on testing access and demand. [97,6] For such 
medRxiv preprint doi: https://doi.org/10.1101/2021.02.11.21251602; this version posted February 12, 2021. The copyright holder for this preprint (which was not certified by peer review) is the author/funder, who has granted medRxiv a license to display the preprint in perpetuity.

It is made available under a CC-BY-NC-ND 4.0 International license .

reasons, the US National Pandemic Strategy called for transitioning from counting individual confirmed cases to monitor epidemic trends to monitoring illness rates (i.e., hospitalization admissions and syndromic surveillance) which are generally less subject to bias. [3] In fact, during the H1N1 pandemic, CDC discontinued state reporting of individual lab-confirmed cases two months after the initiation of the epidemic and initiated state reporting of total numbers of weekly H1N1 hospitalizations and deaths. [5] The CDC used these numbers along other surveillance data to provide monthly estimates of symptomatic illness, hospitalizations, and deaths. [98] During H1N1, CDC also used syndromic surveillance data to model infection dynamics at sub-state regional level, illustrating important regional and temporal patterns of transmission relevant for control and planning decisions. [5]

A robust, national approach to monitoring COVID-19 in every region using multiple measures of disease incidence should be feasible. In July 2020, the US Department of Health and Human Services required all hospitals to report COVID-19 associated admissions to the Federal government as part of a new national reporting standard; this data should allow publishing real time estimates of new hospital admissions by age at a regional level. Coronavirus-like-illness emergency department visits from the NSSP could also be a nationally consistent regional measure. Consistent application of surveillance measures across U.S. regions could lead to better informed and targeted mitigations and their evaluation.

Disaggregating measures of infection and disease burden by community setting (e.g. residential setting, workplaces, neighborhoods) could further aide in targeting public health responses and focusing resources for testing, contact tracing and outbreak investigations. Since May, CMS has required reporting of confirmed cases and deaths from regulated skilled nursing facilities; however, there is still little systematic data on the burden of infection, morbidity, and mortality in other community settings. Through the National Notifiable Disease Surveillance System (NNDSS), CDC has accumulated over 20 million case reports. These reports should contain exposure information on residence, occupation, travel. Analytic studies could combine exposure prevalence among confirmed cases along with population exposure prevalence to estimate attributable risks for each exposure. 
The US currently lacks regional estimates of the cumulative infection prevalence. Regional differences in prevalence could explain part of the variation in the pace of infectious spread. Methods to assess exposure using antibodies have evolved and improved over the epidemic time course. [99,100] Applying serial seroprevalence studies both to estimate cumulative incidence and infection fatality rates should be an important subject of investigation.

\section{Conclusions}

Twelve months after the identification of the SARS-CoV-2 virus, the understanding of COVID-19 transmission, infection severity and disease burden has advanced, yet estimates of several essential epidemiological parameters remain absent or uncertain. Missing are comparative measures of transmission risk and disease burden for community exposure settings, including work in "essential occupations." Estimates for infection fatality and infection hospitalization ratios representative of US settings do not exist. Indicators of disease burden, though available, have insufficient resolution to inform targeted policy and programmatic responses.

These epidemiological data gaps may be limiting the most efficient and equitable response to the COVID19 epidemic and underscore the importance of standardizing data collection priorities and protocols early during a rapidly emerging infectious disease epidemic. CDC scientists and staff have authored a large number of studies on COVID-19; however, the majority are descriptive and provide low-quality evidence for policy and management decisions. The content of their investigations raises questions about whether and how an explicit national research agenda guided CDC epidemiological endeavors.

CDC scientists have the access to data, the expertise, and the resources to provide the data necessary for an optimal epidemic response. Moving forward, the CDC should now plan for how it might develop and implement a timely, strategic, and prioritized national epidemiological data collection and research agenda for the next emerging infectious disease epidemic. 
1 Centers for Disease Control and Prevention. Pandemic Planning Scenarios, Updated: Sept 10, 2020.

2 Mishra S, Kwong,J, Chan A, Baral S

CMAJ June 22, 2020192 (25) E684-E685; DOI: https://doi.org/10.1503/cmaj.201112.

3 Holloway R, Rasmussen SA, Zaza S, Cox NJ, Jernigan DB. Updated preparedness and response framework for influenza pandemics. MMWR Recomm Rep. 2014 Sep 26;63(RR-06):1-18. PMID: 25254666.

4 Reed C, Biggerstaff M, Finelli L, Koonin LM, Beauvais D, Uzicanin A, Plummer A, Bresee J, Redd SC, Jernigan DB. Novel framework for assessing epidemiologic effects of influenza epidemics and pandemics. Emerg Infect Dis. 2013 Jan;19(1):85-91. doi: 10.3201/eid1901.120124. PMID: 23260039; PMCID: PMC3557974.

5 Jhung MA, Swerdlow D, Olsen SJ, Jernigan D, Biggerstaff M, Kamimoto L, Kniss K, Reed C, Fry A, Brammer L, Gindler J, Gregg WJ, Bresee J, Finelli L. Epidemiology of 2009 pandemic influenza A (H1N1) in the United States. Clin Infect Dis. 2011 Jan 1;52 Suppl 1:S13-26. doi: 10.1093/cid/ciq008. PMID: 21342884

6 Stoto MA. The effectiveness of U.S. public health surveillance systems for situational awareness during the 2009 H1N1 pandemic: a retrospective analysis. PLoS One. 2012;7(8):e40984. doi: 10.1371/journal.pone.0040984.

7 Lipsitch M, Riley S, Cauchemez S, Ghani AC, Ferguson NM. Managing and reducing uncertainty in an emerging influenza pandemic. N Engl J Med. 2009 Jul 9;361(2):112-5. doi: 10.1056/NEJMp0904380. Epub 2009 May 27.

PMID: 19474417; PMCID: PMC3066026.

8Improving the evidence base for decision making during a pandemic: the example of 2009 influenza A/H1N1. Biosecur Bioterror. 2011 Jun;9(2):89-115. doi: 10.1089/bsp.2011.0007. PMID: 21612363; PMCID: PMC3102310.

9 Adhikari, S., Meng, S., Wu, YJ. et al. Epidemiology, causes, clinical manifestation and diagnosis, prevention and control of coronavirus disease (COVID-19) during the early outbreak period: a scoping review. Infect Dis Poverty 9 , 29 (2020). https://doi.org/10.1186/s40249-020-00646-x

10 Lipsitch M, Swerdlow DL, Finelli L. Defining the Epidemiology of Covid-19 - Studies Needed. N Engl J Med. 2020 Mar 26;382(13):1194-1196. doi: 10.1056/NEJMp2002125. Epub 2020 Feb 19. PMID: 32074416.

11 O'Brien, K.K., Colquhoun, H., Levac, D. et al. Advancing scoping study methodology: a web-based survey and consultation of perceptions on terminology, definition and methodological steps. BMC Health Serv Res 16, 305 (2016). https://doi.org/10.1186/s12913-016-1579-z

12 Tsang TK, Lau LLH, Cauchemez S, Cowling BJ. Household transmission of influenza virus. Trends Microbiol. 2016;24:123-33.

13 Fine PE. The interval between successive cases of an infectious disease. Am J Epidemiol. 2003 Dec 1;158(11):1039-47. doi: 10.1093/aje/kwg251.

14 Fraser C, Riley S, Anderson RM, Ferguson NM. Factors that make an infectious disease outbreak controllable. Proc Natl Acad Sci U S A. 2004 Apr 20;101(16):6146-51. doi: 10.1073/pnas.0307506101.

15 https://www.cdc.gov/coronavirus/2019-ncov/hep/planning-scenarios.html

16 Li Q, Guan X, Wu P, et al. Early Transmission Dynamics in Wuhan, China, of Novel Coronavirus-Infected Pneumonia. N Engl J Med. 2020;382(13):1199-1207

17 McAloon C, Collins Á, Hunt K, Barber A, Byrne AW, Butler F, Casey M, Griffin J, Lane E, McEvoy D, Wall P, Green M, O'Grady L, More SJ. Incubation period of COVID-19: a rapid systematic review and meta-analysis of observational research. BMJ Open. 2020 Aug 
18 He X, Lau EHY, Wu P, Deng X, Wang J, Hao X, Lau YC, Wong JY, Guan Y, Tan X, Mo X, Chen Y, Liao B, Chen W, Hu F, Zhang Q, Zhong M, Wu Y, Zhao L, Zhang F, Cowling BJ, Li F, Leung GM. Temporal dynamics in viral shedding and transmissibility of COVID-19. Nat Med. 2020 May;26(5):672-675. doi: 10.1038/s41591-0200869-5. Epub 2020 Apr 15. Erratum in: Nat Med. 2020 Sep;26(9):1491-1493.

19 Casey, M., Griffin, J., McAloon, C.G., Byrne, A.W., Madden, J.M., McEvoy, D., Collins, A.B., Hunt, K., Barber, A., Butler, F. and Lane, E.A. (2020). Estimating pre-symptomatic transmission of COVID-19: a secondary analysis using published data. medRxiv.

20 https://www.cdc.gov/coronavirus/2019-ncov/hcp/duration-

isolation.html\#: : text=Available $\% 20$ data $\% 20$ indicate $\% 20$ that $\% 20$ persons, $20 \% 20$ days $\% 20$ after $\% 20$ symptom $\% 20$ on set.

21 Byambasuren, O., Cardona, M., Bell, K., Clark, J., McLaws, M. L., \& Glasziou, P. (2020). Estimating the extent of true asymptomatic COVID-19 and its potential for community transmission: systematic review and metaanalysis. Available at SSRN 3586675

22 Hauser A, Counotte MJ, Margossian CC, Konstantinoudis G, Low N, Althaus CL, Riou J. Estimation of SARSCoV-2 mortality during the early stages of an epidemic: A modeling study in Hubei, China, and six regions in Europe. PLoS Med. 2020 Jul 28;17(7):e1003189. doi: 10.1371/journal.pmed.1003189.

23 https://www.cdc.gov/coronavirus/2019-ncov/covid-data/covid-net/purpose-methods.html

24 https://www.cdc.gov/coronavirus/2019-ncov/covid-data/investigations-discovery/hospitalization-death-byage.html

25 https://www.cdc.gov/nchs/covid19/index.htm

26 https://www.cdc.gov/coronavirus/2019-ncov/covid-data/investigations-discovery/hospitalization-death-by-raceethnicity.html

27 https://www.cdc.gov/nchs/nvss/vsrr/covid19/index.htm

28 https://www.cdc.gov/coronavirus/2019-ncov/covid-data/covidview/index.html\#outpatient

29 https://gis.cdc.gov/grasp/fluview/main.html

30 https://covid.cdc.gov/covid-data-tracker/\#ed-visits

31 https://www.cdc.gov/nssp/

32 https://gis.cdc.gov/grasp/COVIDNet/COVID19 3.html

33 https://www.cdc.gov/coronavirus/2019-ncov/covid-data/covidview/index.html

34 https://www.cdc.gov/nchs/nvss/covid-19.htm

35 https://covid.cdc.gov/covid-data-tracker/\#national-lab

36 https://www.cdc.gov/coronavirus/2019-ncov/cases-updates/burden.html

37 Centers for Disease Control and Prevention. Pandemic Planning Scenarios, Updated: May 20, 2020 
38 Centers for Disease Control and Prevention. https://www.cdc.gov/coronavirus/2019-ncov/coviddata/investigations-discovery/assessing-risk-factors.html.

39 Centers for Disease Control and Prevention. COVID-NET. https://gis.cdc.gov/grasp/covidnet/COVID19_3.html.

40 Centers for Disease Control and Prevention. National Center for Health Statistics Provisional COVID-19 Death Counts. https://www.cdc.gov/nchs/nvss/vsrr/covid_weekly/index.htm.

41 Havers FP, Reed C, Lim T, Montgomery JM, Klena JD, Hall AJ, Fry AM, Cannon DL, Chiang CF, Gibbons A, Krapiunaya I, Morales-Betoulle M, Roguski K, Rasheed MAU, Freeman B, Lester S, Mills L, Carroll DS, Owen SM, Johnson JA, Semenova V, Blackmore C, Blog D, Chai SJ, Dunn A, Hand J, Jain S, Lindquist S, Lynfield R, Pritchard S, Sokol T, Sosa L, Turabelidze G, Watkins SM, Wiesman J, Williams RW, Yendell S, Schiffer J, Thornburg NJ. Seroprevalence of Antibodies to SARS-CoV-2 in 10 Sites in the United States, March 23-May 12, 2020. JAMA Intern Med. 2020 Jul 21. doi: 10.1001/jamainternmed.2020.4130. Epub ahead of print. PMID: 32692365 .

42 Reese H, Iuliano A, Patel N, Garg S, Kim L, Silk B, Hall A, Fry A, Reed, C. Estimated incidence of COVID-19 illness and hospitalization - United States, February-September, 2020, Clinical Infectious Diseases, , ciaa1780, https://doi.org/10.1093/cid/ciaa1780.

43 Garg S, Kim L, Whitaker M, O'Halloran A, Cummings C, Holstein R, Prill M, Chai SJ, Kirley PD, Alden NB, Kawasaki B, Yousey-Hindes K, Niccolai L, Anderson EJ, Openo KP, Weigel A, Monroe ML, Ryan P, Henderson J, Kim S, Como-Sabetti K, Lynfield R, Sosin D, Torres S, Muse A, Bennett NM, Billing L, Sutton M, West N, Schaffner W, Talbot HK, Aquino C, George A, Budd A, Brammer L, Langley G, Hall AJ, Fry A. Hospitalization Rates and Characteristics of Patients Hospitalized with Laboratory-Confirmed Coronavirus Disease 2019 - COVIDNET, 14 States, March 1-30, 2020. MMWR Morb Mortal Wkly Rep. 2020 Apr 17;69(15):458-464. doi: 10.15585/mmwr.mm6915e3. PMID: 32298251.

44 Mosites E, Parker EM, Clarke KEN, Gaeta JM, Baggett TP, Imbert E, Sankaran M, Scarborough A, Huster K, Hanson M, Gonzales E, Rauch J, Page L, McMichael TM, Keating R, Marx GE, Andrews T, Schmit K, Morris SB, Dowling NF, Peacock G; COVID-19 Homelessness Team. Assessment of SARS-CoV-2 Infection Prevalence in Homeless Shelters - Four U.S. Cities, March 27-April 15, 2020. MMWR Morb Mortal Wkly Rep. 2020 May 1;69(17):521-522. doi: 10.15585/mmwr.mm6917e1. PMID: 32352957; PMCID: PMC7206983.

45 New York City Department of Mental Health and Hygiene Covid-19 Response Team. Preliminary Estimate of Excess Mortality During the COVID-19 Outbreak - New York City, March 11-May 2, 2020. MMWR Morb Mortal Wkly Rep 2020;69:603-605. DOI: http://dx.doi.org/10.15585/mmwr.mm6919e5external icon

46 Jorden MA, Rudman SL, et al. Evidence for Limited Early Spread of COVID-19 Within the United States, January-February 2020. MMWR Morb Mortal Wkly Rep 2020;69:680-684.

DOI: http://dx.doi.org/10.15585/mmwr.mm6922elexternal icon

47 Bushman D, Alroy KA, Greene SK, et al. Detection and Genetic Characterization of Community-Based SARSCoV-2 Infections — New York City, March 2020. MMWR Morb Mortal Wkly Rep 2020;69:918-922.

DOI: http://dx.doi.org/10.15585/mmwr.mm6928a5

48 Biggs HM, Harris JB, Breakwell L, Dahlgren FS, Abedi GR, Szablewski CM, Drobeniuc J, Bustamante ND, Almendares O, Schnall AH, Gilani Z, Smith T, Gieraltowski L, Johnson JA, Bajema KL, McDavid K, Schafer IJ, Sullivan V, Punkova L, Tejada-Strop A, Amiling R, Mattison CP, Cortese MM, Ford SE, Paxton LA, Drenzek C, Tate JE; CDC Field Surveyor Team. Estimated Community Seroprevalence of SARS-CoV-2 Antibodies - Two Georgia Counties, April 28-May 3, 2020. MMWR Morb Mortal Wkly Rep. 2020 Jul 24;69(29):965-970. doi: 10.15585/mmwr.mm6929e2. PMID: 32701941; PMCID: PMC7377817.

49 Menachemi N, Yiannoutsos CT, Dixon BE, Duszynski TJ, Fadel WF, Wools-Kaloustian KK, Unruh Needleman N, Box K, Caine V, Norwood C, Weaver L, Halverson PK. Population Point Prevalence of SARS-CoV-2 Infection 
Based on a Statewide Random Sample - Indiana, April 25-29, 2020. MMWR Morb Mortal Wkly Rep. 2020 Jul 24;69(29):960-964. doi: 10.15585/mmwr.mm6929e1. PMID: 32701938; PMCID: PMC7377824

50 Sutton M, Cieslak P, Linder M. Notes from the Field: Seroprevalence Estimates of SARS-CoV-2 Infection in Convenience Sample — Oregon, May 11-June 15, 2020. MMWR Morb Mortal Wkly Rep 2020;69:1100-1101. DOI: http://dx.doi.org/10.15585/mmwr.mm6932a4

51 Hatfield KM, Reddy SC, Forsberg K, et al. Facility-Wide Testing for SARS-CoV-2 in Nursing Homes - Seven U.S. Jurisdictions, March-June 2020. MMWR Morb Mortal Wkly Rep 2020;69:1095-1099.

DOI: http://dx.doi.org/10.15585/mmwr.mm6932e5external icon

52 Kim L, Whitaker M, O'Halloran A, Kambhampati A, Chai SJ, Reingold A, Armistead I, Kawasaki B, Meek J, Yousey-Hindes K, Anderson EJ, Openo KP, Weigel A, Ryan P, Monroe ML, Fox K, Kim S, Lynfield R, Bye E, Shrum Davis S, Smelser C, Barney G, Spina NL, Bennett NM, Felsen CB, Billing LM, Shiltz J, Sutton M, West N, Talbot HK, Schaffner W, Risk I, Price A, Brammer L, Fry AM, Hall AJ, Langley GE, Garg S; COVID-NET Surveillance Team. Hospitalization Rates and Characteristics of Children Aged $<18$ Years Hospitalized with Laboratory-Confirmed COVID-19 - COVID-NET, 14 States, March 1-July 25, 2020. MMWR Morb Mortal Wkly Rep. 2020 Aug.

53 Hagan LM, Williams SP, Spaulding AC, et al. Mass Testing for SARS-CoV-2 in 16 Prisons and Jails - Six Jurisdictions, United States, April-May 2020. MMWR Morb Mortal Wkly Rep 2020;69:1139-1143.

DOI: http://dx.doi.org/10.15585/mmwr.mm6933a3external icon

54 McBee SM, Thomasson ED, Scott MA, et al. Notes from the Field: Universal Statewide Laboratory Testing for SARS-CoV-2 in Nursing Homes - West Virginia, April 21-May 8, 2020. MMWR Morb Mortal Wkly Rep 2020;69:1177-1179. DOI: http://dx.doi.org/10.15585/mmwr.mm6934a4

55 Self WH, Tenforde MW, Stubblefield WB, et al. Seroprevalence of SARS-CoV-2 Among Frontline Health Care Personnel in a Multistate Hospital Network - 13 Academic Medical Centers, April-June 2020. MMWR Morb Mortal Wkly Rep 2020;69:1221-1226. DOI: http://dx.doi.org/10.15585/mmwr.mm6935e2

56 Bixler D, Miller AD, Mattison CP, et al. SARS-CoV-2-Associated Deaths Among Persons Aged $<21$ Years United States, February 12-July 31, 2020. MMWR Morb Mortal Wkly Rep 2020;69:1324-1329.

DOI: http://dx.doi.org/10.15585/mmwr.mm6937e4external icon

57 Rossen LM, Branum AM, Ahmad FB, Sutton P, Anderson RN. Excess Deaths Associated with COVID-19, by Age and Race and Ethnicity - United States, January 26-October 3, 2020. MMWR Morb Mortal Wkly Rep 2020;69:1522-1527. DOI: http://dx.doi.org/10.15585/mmwr.mm6942e2

58 Burke RM, Midgley CM, Dratch A, Fenstersheib M, Haupt T, Holshue M, Ghinai I, Jarashow MC, Lo J, McPherson TD, Rudman S, Scott S, Hall AJ, Fry AM, Rolfes MA. Active Monitoring of Persons Exposed to Patients with Confirmed COVID-19 - United States, January-February 2020. MMWR Morb Mortal Wkly Rep. 2020 Mar 6;69(9):245-246. doi: 10.15585/mmwr.mm6909e1. PMID: 32134909; PMCID: PMC7367094.

59 Lewis NM, Chu VT, Ye D, Conners EE, Gharpure R, Laws RL, Reses HE, Freeman BD, Fajans M, Rabold EM, Dawson P, Buono S, Yin S, Owusu D, Wadhwa A, Pomeroy M, Yousaf A, Pevzner E, Njuguna H, Battey KA, Tran CH, Fields VL, Salvatore P, O'Hegarty M, Vuong J, Chancey R, Gregory C, Banks M, Rispens JR, Dietrich E, Marcenac P, Matanock AM, Duca L, Binder A, Fox G, Lester S, Mills L, Gerber SI, Watson J, Schumacher A, Pawloski L, Thornburg NJ, Hall AJ, Kiphibane T, Willardson S, Christensen K, Page L, Bhattacharyya S, Dasu T, Christiansen A, Pray IW, Westergaard RP, Dunn AC, Tate JE, Nabity SA, Kirking HL. Household Transmission of SARS-CoV-2 in the United States. Clin Infect Dis. 2020 Aug 16:ciaa1166. doi: 10.1093/cid/ciaa1166. Epub ahead of print. PMID: 33185244; PMCID: PMC7454394.

60 Grijalva CG, Rolfes MA, Zhu Y, et al. Transmission of SARS-COV-2 Infections in Households - Tennessee and Wisconsin, April-September 2020. MMWR Morb Mortal Wkly Rep 2020;69:1631-1634.

DOI: http://dx.doi.org/10.15585/mmwr.mm6944elexternal icon 
61 Rosenberg ES, Dufort EM, Blog DS, Hall EW, Hoefer D, Backenson BP, Muse AT, Kirkwood JN, St George K, Holtgrave DR, Hutton BJ, Zucker HA; New York State Coronavirus 2019 Response Team. COVID-19 Testing, Epidemic Features, Hospital Outcomes, and Household Prevalence, New York State-March 2020. Clin Infect Dis. 2020 Nov 5;71(8):1953-1959. doi: 10.1093/cid/ciaa549. PMID: 32382743 ; PMCID: PMC7239264.

62 Payne DC, Smith-Jeffcoat SE, Nowak G, Chukwuma U, Geibe JR, Hawkins RJ, Johnson JA, Thornburg NJ, Schiffer J, Weiner Z, Bankamp B, Bowen MD, MacNeil A, Patel MR, Deussing E; CDC COVID-19 Surge Laboratory Group, Gillingham BL. SARS-CoV-2 Infections and Serologic Responses from a Sample of U.S. Navy Service Members - USS Theodore Roosevelt, April 2020. MMWR Morb Mortal Wkly Rep. 2020 Jun 12;69(23):714-721. doi: 10.15585/mmwr.mm6923e4. PMID: 32525850; PMCID: PMC7315794.

63 Lewis NM, Friedrichs M, Wagstaff S, Sage K, LaCross N, Bui D, McCaffrey K, Barbeau B, George A, Rose C, Willardson S, Carter A, Smoot C, Nakashima A, Dunn A. Disparities in COVID-19 Incidence, Hospitalizations, and Testing, by Area-Level Deprivation - Utah, March 3-July 9, 2020. MMWR Morb Mortal Wkly Rep. 2020 Sep 25;69(38):1369-1373. doi: 10.15585/mmwr.mm6938a4. PMID: 32970656.

64 Bui DP, McCaffrey K, Friedrichs M, LaCross N, Lewis NM, Sage K, Barbeau B, Vilven D, Rose C, Braby S, Willardson S, Carter A, Smoot C, Winquist A, Dunn A. Racial and Ethnic Disparities Among COVID-19 Cases in Workplace Outbreaks by Industry Sector - Utah, March 6-June 5, 2020. MMWR Morb Mortal Wkly Rep. 2020 Aug 21;69(33):1133-1138. doi: 10.15585/mmwr.mm6933e3. PMID: 32817604; PMCID: PMC7439983.

65 Bui DP, See I, Hesse EM, Varela K, Harvey RR, August EM, Winquist A, Mullins S, McBee S, Thomasson E, Atkins A. Association Between CMS Quality Ratings and COVID-19 Outbreaks in Nursing Homes - West Virginia, March 17-June 11, 2020. MMWR Morb Mortal Wkly Rep. 2020 Sep 18;69(37):1300-1304. doi:

10.15585/mmwr.mm6937a5. PMID: 32941409; PMCID: PMC7498166.

66 Fisher KA, Tenforde MW, Feldstein LR, Lindsell CJ, Shapiro NI, Files DC, Gibbs KW, Erickson HL, Prekker ME, Steingrub JS, Exline MC, Henning DJ, Wilson JG, Brown SM, Peltan ID, Rice TW, Hager DN, Ginde AA, Talbot HK, Casey JD, Grijalva CG, Flannery B, Patel MM, Self WH; IVY Network Investigators; CDC COVID-19 Response Team. Community and Close Contact Exposures Associated with COVID-19 Among Symptomatic Adults $\geq 18$ Years in 11 Outpatient Health Care Facilities - United States, July 2020. MMWR Morb Mortal Wkly Rep. 2020 Sep 11;69(36):1258-1264. doi: 10.15585/mmwr.mm6936a5. PMID: 32915165; PMCID: PMC7499837.

67 Telford CT, Onwubiko U, Holland DP, Turner K, Prieto J, Smith S, Yoon J, Brown W, Chamberlain A, Gandhi N, Williams S, Khan F, Shah S. Preventing COVID-19 Outbreaks in Long-Term Care Facilities Through Preemptive Testing of Residents and Staff Members - Fulton County, Georgia, March-May 2020. MMWR Morb Mortal Wkly Rep. 2020 Sep 18;69(37):1296-1299. doi: 10.15585/mmwr.mm6937a4. PMID: 32941413; PMCID: PMC7498169.

68 Dasgupta S, Bowen VB, Leidner A, et al. Association Between Social Vulnerability and a County's Risk for Becoming a COVID-19 Hotspot - United States, June 1-July 25, 2020. MMWR Morb Mortal Wkly Rep 2020;69:1535-1541. DOI: http://dx.doi.org/10.15585/mmwr.mm6942a3external icon

69 Yoon JC, Montgomery MP, Buff AM, Boyd AT, Jamison C, Hernandez A, Schmit K, Shah S, Ajoku S, Holland DP, Prieto J, Smith S, Swancutt MA, Turner K, Andrews T, Flowers K, Wells A, Marchman C, Laney E, Bixler D, Cavanaugh S, Flowers N, Gaffga N, Ko JY, Paulin HN, Weng MK, Mosites E, Morris SB. COVID-19 Prevalence among People Experiencing Homelessness and Homelessness Service Staff during Early Community Transmission in Atlanta, Georgia, April-May 2020. Clin Infect Dis. 2020 Sep 8:ciaa1340. doi: 10.1093/cid/ciaa1340. Epub ahead of print. PMID: 32898272; PMCID: PMC7499502.

70 Teran RA, Ghinai I, Gretsch S, et al. COVID-19 Outbreak Among a University's Men's and Women's Soccer Teams - Chicago, Illinois, July-August 2020. MMWR Morb Mortal Wkly Rep 2020;69:1591-1594. DOI: http://dx.doi.org/10.15585/mmwr.mm6943e5external icon

71 CDC COVID-19 Response Team. Preliminary Estimates of the Prevalence of Selected Underlying Health Conditions Among Patients with Coronavirus Disease 2019 - United States, February 12-March 28, 2020. MMWR 
Morb Mortal Wkly Rep. 2020 Apr 3;69(13):382-386. doi: 10.15585/mmwr.mm6913e2. PMID: 32240123; PMCID: PMC7119513.

72 Ellington S, Strid P, Tong VT, Woodworth K, Galang RR, Zambrano LD, Nahabedian J, Anderson K, Gilboa SM. Characteristics of Women of Reproductive Age with Laboratory-Confirmed SARS-CoV-2 Infection by Pregnancy Status - United States, January 22-June 7, 2020. MMWR Morb Mortal Wkly Rep. 2020 Jun 26;69(25):769-775. doi: 10.15585/mmwr.mm6925a1. PMID: 32584795; PMCID: PMC7316319.

73 Killerby ME, Link-Gelles R, Haight SC, Schrodt CA, England L, Gomes DJ, Shamout M, Pettrone K, O'Laughlin K, Kimball A, Blau EF, Burnett E, Ladva CN, Szablewski CM, Tobin-D'Angelo M, Oosmanally N, Drenzek C, Murphy DJ, Blum JM, Hollberg J, Lefkove B, Brown FW, Shimabukuro T, Midgley CM, Tate JE; CDC COVID-19 Response Clinical Team. Characteristics Associated with Hospitalization Among Patients with COVID19 - Metropolitan Atlanta, Georgia, March-April 2020. MMWR Morb Mortal Wkly Rep. 2020 Jun 26;69(25):790794. doi: 10.15585/mmwr.mm6925e1. PMID: 32584797; PMCID: PMC7316317.

74 Tenforde MW, Kim SS, Lindsell CJ, Billig Rose E, Shapiro NI, Files DC, Gibbs KW, Erickson HL, Steingrub JS, Smithline HA, Gong MN, Aboodi MS, Exline MC, Henning DJ, Wilson JG, Khan A, Qadir N, Brown SM, Peltan ID, Rice TW, Hager DN, Ginde AA, Stubblefield WB, Patel MM, Self WH, Feldstein LR; IVY Network Investigators; CDC COVID-19 Response Team; IVY Network Investigators. Symptom Duration and Risk Factors for Delayed Return to Usual Health Among Outpatients with COVID-19 in a Multistate Health Care Systems Network - United States, March-June 2020. MMWR Morb Mortal Wkly Rep. 2020 Jul 31;69(30):993-998. doi: 10.15585/mmwr.mm6930e1. PMID: 32730238; PMCID: PMC7392393.

75 Jackson BR, Gold JAW, Natarajan P, Rossow J, Neblett Fanfair R, da Silva J, Wong KK, Browning SD, Bamrah Morris S, Rogers-Brown J, Hernandez-Romieu AC, Szablewski CM, Oosmanally N, Tobin-D'Angelo M, Drenzek C, Murphy DJ, Hollberg J, Blum JM, Jansen R, Wright DW, SeweSll WM, Owens JD, Lefkove B, Brown FW, Burton DC, Uyeki TM, Bialek SR, Patel PR, Bruce BB. Predictors at admission of mechanical ventilation and death in an observational cohort of adults hospitalized with COVID-19. Clin Infect Dis. 2020 Sep 24:ciaa1459. doi: 10.1093/cid/ciaa1459. Epub ahead of print. PMID: 32971532; PMCID: PMC7543323.

76 Yousaf A, Duca L, Chu V et al.A Prospective Cohort Study in Nonhospitalized Household Contacts With Severe Acute Respiratory Syndrome Coronavirus 2 Infection: Symptom Profiles and Symptom Change Over Time, Clinical Infectious Diseases, , ciaa1072, https://doi.org/10.1093/cid/ciaa1072

77 Dawson P, Rabold EM, Laws RL, Conners EE, Gharpure R, Yin S, Buono SA, Dasu T, Bhattacharyya S, Westergaard RP, Pray IW, Ye D, Nabity SA, Tate JE, Kirking HL. Loss of Taste and Smell as Distinguishing Symptoms of COVID-19. Clin Infect Dis. 2020 Jun 21:ciaa799. doi: 10.1093/cid/ciaa799. Epub ahead of print. PMID: 32562541; PMCID: PMC7337666.

78 Santoli JM, Lindley MC, DeSilva MB, et al. Effects of the COVID-19 Pandemic on Routine Pediatric Vaccine Ordering and Administration — United States, 2020. MMWR Morb Mortal Wkly Rep 2020;69:591-593. DOI: http://dx.doi.org/10.15585/mmwr.mm6919e2

79 Bramer CA, Kimmins LM, Swanson R, et al. Decline in Child Vaccination Coverage During the COVID-19 Pandemic - Michigan Care Improvement Registry, May 2016-May 2020. MMWR Morb Mortal Wkly Rep 2020;69:630-631. DOI: http://dx.doi.org/10.15585/mmwr.mm6920e1

80 Hartnett KP, Kite-Powell A, DeVies J, Coletta MA, Boehmer TK, Adjemian J, Gundlapalli AV; National Syndromic Surveillance Program Community of Practice. Impact of the COVID-19 Pandemic on Emergency Department Visits - United States, January 1, 2019-May 30, 2020. MMWR Morb Mortal Wkly Rep. 2020 Jun 12;69(23):699-704. doi: 10.15585/mmwr.mm6923e1. PMID: 32525856; PMCID: PMC7315789.

81 Lange SJ, Ritchey MD, Goodman AB, et al. Potential Indirect Effects of the COVID-19 Pandemic on Use of Emergency Departments for Acute Life-Threatening Conditions — United States, January-May 2020. MMWR Morb Mortal Wkly Rep 2020;69:795-800. DOI: http://dx.doi.org/10.15585/mmwr.mm6925e2 
82 Groenewold MR, Burrer SL, Ahmed F, Uzicanin A, Free H, Luckhaupt SE. Increases in Health-Related Workplace Absenteeism Among Workers in Essential Critical Infrastructure Occupations During the COVID-19 Pandemic - United States, March-April 2020. MMWR Morb Mortal Wkly Rep. 2020 Jul 10;69(27):853-858. doi: 10.15585/mmwr.mm6927a1. PMID: 32644979.

83 Langdon-Embry M, Papadouka V, Cheng I, Almashhadani M, Ternier A, Zucker JR. Notes from the Field: Rebound in Routine Childhood Vaccine Administration Following Decline During the COVID-19 Pandemic — New York City, March 1-June 27, 2020. MMWR Morb Mortal Wkly Rep 2020;69:999-1001. DOI: http://dx.doi.org/10.15585/mmwr.mm6930a3external icon

84 Czeisler MÉ, Marynak K, Clarke KE, et al. Delay or Avoidance of Medical Care Because of COVID-19-Related Concerns - United States, June 2020. MMWR Morb Mortal Wkly Rep 2020;69:1250-1257. DOI: http://dx.doi.org/10.15585/mmwr.mm6936a4external icon

85 Czeisler MÉ, Lane RI, Petrosky E, Wiley JF, Christensen A, Njai R, Weaver MD, Robbins R, Facer-Childs ER, Barger LK, Czeisler CA, Howard ME, Rajaratnam SMW. Mental Health, Substance Use, and Suicidal Ideation During the COVID-19 Pandemic - United States, June 24-30, 2020. MMWR Morb Mortal Wkly Rep. 2020 Aug 14;69(32):1049-1057. doi: 10.15585/mmwr.mm6932a1. PMID: 32790653; PMCID: PMC7440121.

86 Koonin LM, Hoots B, Tsang CA, et al. Trends in the Use of Telehealth During the Emergence of the COVID-19 Pandemic - United States, January-March 2020. MMWR Morb Mortal Wkly Rep 2020;69:1595-1599. DOI: http://dx.doi.org/10.15585/mmwr.mm6943a3

87 Oran DP, Topol EJ. Prevalence of Asymptomatic SARS-CoV-2 Infection : A Narrative Review. Ann Intern Med. 2020 Sep 1;173(5):362-367. doi: 10.7326/M20-3012. Epub 2020 Jun 3. PMID: 32491919; PMCID: PMC7281624.

88 Davies NG, Klepac P, Liu Y, Prem K, Jit M. Age-dependent effects in the transmission and control of COVID19 epidemics. Nat Med. 2020Jun 16;26(8). doi:10.1038/s41591-020-0962-9

89 Poletti, P., Tirani, M., Cereda, D., Trentini, F., Guzzetta, G., Sabatino, G., Marziano, V., Castrofino, A., Grosso, F., Del Castillo, G. and Piccarreta, R. (2020). Probability of symptoms and critical disease after SARS-CoV-2 infection. arXiv preprint: https://arxiv.org/abs/2006.08471.

90 Buitrago-Garcia D, Egli-Gany D, Counotte MJ, Hossmann S, Imeri H, Ipekci AM, Salanti G, Low N. Occurrence and transmission potential of asymptomatic and presymptomatic SARS-CoV-2 infections: A living systematic review and meta-analysis. PLoS Med. 2020 Sep 22;17(9):e1003346. doi: 10.1371/journal.pmed.1003346.

91 Honein MA, Christie A, Rose DA, et al. Summary of Guidance for Public Health Strategies to Address High Levels of Community Transmission of SARS-CoV-2 and Related Deaths, December 2020. MMWR Morb Mortal Wkly Rep. ePub: 4 December 2020. DOI: http://dx.doi.org/10.15585/mmwr.mm6949e2

92 Cauchemez S, Donnelly CA, Reed C, Ghani AC, Fraser C, Kent CK, Finelli L, Ferguson NM. Household transmission of 2009 pandemic influenza A (H1N1) virus in the United States. N Engl J Med. 2009 Dec 31;361(27):2619-27. doi: 10.1056/NEJMoa0905498. PMID: 20042753; PMCID: PMC3840270.

93 Fung H, Martinez L, Alarid-Escudero F, et al. Stanford-CIDE Coronavirus Simulation Model (SC-COSMO) Modeling Group, The Household Secondary Attack Rate of Severe Acute Respiratory Syndrome Coronavirus 2 (SARS-CoV-2): A Rapid Review, Clinical Infectious Diseases, , ciaa1558, https://doi.org/10.1093/cid/ciaa1558

94 Glatman-Freedman A, Portelli I, Jacobs SK, Mathew JI, Slutzman JE, Goldfrank LR, Smith SW. Attack rates assessment of the 2009 pandemic H1N1 influenza A in children and their contacts: a systematic review and metaanalysis. PLoS One. 2012;7(11):e50228. doi: 10.1371/journal.pone.0050228. Epub 2012 Nov 30. PMID: 23284603; PMCID: PMC3523802.

95 Centers for Disease Control and Prevention. https://www.cdc.gov/coronavirus/2019-ncov/coviddata/investigations-discovery/assessing-risk-factors.html. 
96 Schneider EC. Failing the Test - The Tragic Data Gap Undermining the U.S. Pandemic Response. N Engl J Med. 2020 Jul 23;383(4):299-302. doi: 10.1056/NEJMp2014836. Epub 2020 May 15. PMID: 32412704.

97 Lipsitch M, Hayden FG, Cowling BJ, Leung GM. How to maintain surveillance for novel influenza A H1N1 when there are too many cases to count. Lancet. 2009 Oct 3;374(9696):1209-11. doi: 10.1016/S01406736(09)61377-5. Epub 2009 Aug 11. PMID: 19679345.

98 Shrestha SS, Swerdlow DL, Borse RH, Prabhu VS, Finelli L, Atkins CY, Owusu-Edusei K, Bell B, Mead PS, Biggerstaff M, Brammer L, Davidson H, Jernigan D, Jhung MA, Kamimoto LA, Merlin TL, Nowell M, Redd SC, Reed C, Schuchat A, Meltzer MI. Estimating the burden of 2009 pandemic influenza A (H1N1) in the United States (April 2009-April 2010). Clin Infect Dis. 2011 Jan 1;52 Suppl 1:S75-82. doi: 10.1093/cid/ciq012. PMID: 21342903.

99 National SARS-CoV-2 Serology Assay Evaluation Group. Performance characteristics of five immunoassays for SARS-CoV-2: a head-to-head benchmark comparison. Lancet Infect Dis. 2020 Dec;20(12):1390-1400. doi: 10.1016/S1473-3099(20)30634-4. Epub 2020 Sep 23. Erratum in: Lancet Infect Dis. 2020 Dec;20(12):e298. PMID: 32979318; PMCID: PMC7511171.

100 Choe PG, Kang CK, Suh HJ, Jung J, Kang E, Lee SY, Song KH, Kim HB, Kim NJ, Park WB, Kim ES, Oh MD. Antibody Responses to SARS-CoV-2 at 8 Weeks Postinfection in Asymptomatic Patients. Emerg Infect Dis. 2020 Oct;26(10):2484-2487. doi: 10.3201/eid2610.202211. Epub 2020 Jun 24. PMID: 32579877; PMCID: PMC7510710. 